\title{
Singularity Analysis for a Class of Porous Medium Equation with Time-Dependent Coefficients
}

\author{
Anyin Xia, ${ }^{1,2}$ Xianxiang $\mathrm{Pu}^{3}$ and Shan $\mathrm{Li}^{4}$ \\ ${ }^{1}$ Department of Mathematics, Sichuan University, Chengdu 610065, China \\ ${ }^{2}$ School of Science, Xihua University, Chengdu 610039, China \\ ${ }^{3}$ Sichuan Sanhe College of Professionals, Luzhou 646200, China \\ ${ }^{4}$ Business School, Sichuan University, Chengdu 610064, China \\ Correspondence should be addressed to Shan Li; lishan@scu.edu.cn
}

Received 22 September 2015; Accepted 24 December 2015

Academic Editor: Andrei D. Mironov

Copyright (C) 2016 Anyin Xia et al. This is an open access article distributed under the Creative Commons Attribution License, which permits unrestricted use, distribution, and reproduction in any medium, provided the original work is properly cited.

\begin{abstract}
This paper concerns the singularity and global regularity for the porous medium equation with time-dependent coefficients under homogeneous Dirichlet boundary conditions. Firstly, some global regularity results are established. Furthermore, we investigate the blow-up solution to the boundary value problem. The upper and lower estimates to the lifespan of the singular solution are also obtained here.
\end{abstract}

\section{Introduction and Main Results}

In this work, we consider the following porous medium equation with time-dependent coefficients under homogeneous Dirichlet boundary condition:

$$
\begin{aligned}
u_{t} & =\Delta u^{m}+f(t) g(u), \quad(x, t) \in \Omega \times(0, T), \\
u(x, t) & =0, \quad(x, t) \in \partial \Omega \times(0, T), \\
u(x, 0) & =u_{0}(x), \quad x \in \Omega,
\end{aligned}
$$

where $\Omega \subset \mathbb{R}^{N}$ is a smooth bounded domain, $m>1$. The coefficient $f(t)$ is a positive bounded continuous function with $f(t) \leq \bar{k}$ for any $t>0$. The nonlinearity $g(u)$ is assumed to satisfy $g(u)>0(u>0)$ and $g(0)=0$. The initial value $u_{0}(x)$ is nontrivial nonnegative continuous function and vanishes on $\partial \Omega$.

Global existence and nonexistence to the nonlinear parabolic equation are important topic and have been investigated extensively; please see the surveys [1-4]. The first purpose in this paper is to investigate the sufficient conditions to the global existence of the solution to the boundary value problem (1). The second aim of this paper is to investigate the solution which blows up in finite time and estimate the lifespan of the singular solution. Singularity analysis, especially, to evaluate the lifespan of the singular solution is also an interesting research topic in this field.

In [5-7], Payne and others have considered the linear diffusion case. However, the degenerate diffusion makes the present problem more complicated and takes more essential difficulties here. We would like to refer some results on blow-up solutions to the degenerate parabolic equations and system as follows. Some global existence and nonexistence of the classical solution to degenerate parabolic equations were established in [8], and then $\mathrm{Du}$ and his colleagues gave the sufficient conditions to the degenerate parabolic system with nonlinear nonlocal sources in [9-11], with nonlinear localized sources in [10], and with nonlinear memory terms in [12]. Furthermore, some properties to the singular solutions, such as blow-up set, uniform blow-up profile were obtained in [1315].

The local existence of classical solution to system (1) can be obtained by the standard method in [16]. Firstly, we give some results on the global existence of the classical solution to the boundary value problem (1) as follows.

Theorem 1. Suppose that there exists a positive constant $p<$ $m$, such that $g(u) \leq u^{p}$ for $u>0$; then every classical solution to problem (1) is global. 
Secondly, we give the blow-up results in the next theorem.

Theorem 2. Suppose that there exists a positive constant $p$ with $p \geq m$, such that $g(u) \geq u^{p}$ for $u>0$ and $\underline{k}:=\inf f(t)>0$; then the classical solution to problem (1) blows up in finite time, provided that the initiate data are sufficiently large.

Theorem 3. Suppose that there exists a positive constant $p$ with $p \geq m$, such that $g(u) \geq u^{p}$ for $u>0$ and $\underline{k}:=\inf f(t)>$ $\lambda_{1}$; then the classical solution to problem (1) blows up in finite time $T$ for large data $u_{0}(x)$, where $\lambda_{1}$ is the first eigenvalue to the following problem:

$$
\begin{aligned}
\Delta \varphi+\lambda \varphi & =0, \quad x \in \Omega, \\
\varphi & =0, \quad x \in \partial \Omega,
\end{aligned}
$$

with $\varphi>0(x \in \Omega)$ and $\int_{\Omega} \varphi d x=1$. Moreover, there exists a constant $\bar{T}_{0}$, which depends on $m, f, g, u_{0}(x)$, such that $T \leq \bar{T}_{0}$.

Furthermore, we give the following estimates to the maximal blow-up time $T$.

Theorem 4. Suppose that $\Omega$ be a convex domain in $\mathbb{R}^{3}$, and there exists a constant $p>0$, such that $g(u) \leq u^{p}, u>0$. If the solution to problem (1) blows up in finite time $T$, then there exists a positive constant $\underline{T}_{0}$, which depends on $m, f, g, u_{0}(x)$, such that

$$
T \geq \underline{T}_{0} .
$$

Remark 5. We would like to mention that the results in Theorem 4 are still valid for two-dimensional case.

The remainder of this paper is organized as follows. Global existence of the solution to problem (1) is established in Section 2, by constructing some global upper solution. In Sections 3 and 4, we show that the classical solution to problem (1) will blow up in finite time and obtain upper bound of the blow-up time. In the last section, with aid of a differential inequality, we will establish lower estimate to the maximal blow-up time.

\section{Global Solution for Problem (1)}

In this section, we focus on the global solution of (1) and show Theorem 1.

Proof. Obviously, if $0<p<m$, then there exists a positive constant $\sigma$ such that $0<m \sigma<1, m \sigma-p \sigma>0$.

Next, we construct a supersolution which is bounded for any $T>0$. Let $\phi(x)$ be the solution of the following elliptic problem:

$$
\begin{aligned}
-\Delta \phi(x) & =1, \quad x \in \Omega, \\
\phi(x) & =0, \quad x \in \partial \Omega .
\end{aligned}
$$

Denote $C=\max _{\bar{\Omega}} \phi(x)$. Namely, $0 \leq \phi(x) \leq C$.

We define the function $\bar{u}(x, t)$ as

$$
\bar{u}=(K(\phi(x)+1))^{\sigma}
$$

where $\sigma>0$ satisfy $m \sigma<1$ and $K>0$ will be fixed later. Clearly, $\bar{u}$ is bounded for any $t>0$. Thus, we have

$$
\begin{aligned}
\bar{u}_{t} & -\Delta \bar{u}^{m} \\
& =-m \sigma K^{m \sigma}\left\{(m \sigma-1)[1+\phi(x)]^{m \sigma-2}|\nabla \phi(x)|^{2}\right. \\
& \left.+[1+\phi(x)]^{m \sigma-1} \Delta \phi(x)\right\} \geq-m \sigma K^{m \sigma}[1 \\
& +\phi(x)]^{m \sigma-1} \Delta \phi(x)=m \sigma K^{m \sigma}[1+\phi(x)]^{m \sigma-1} \\
& \geq m \sigma K^{m \sigma}(1+C)^{m \sigma-1}, \\
f( & t) g(\bar{u}) \leq \bar{k} \bar{u}^{p} \leq \bar{k}[K(1+\phi(x))]^{p \sigma} \\
& \leq \bar{k}[K(1+C)]^{p \sigma} .
\end{aligned}
$$

Denote

$$
K_{1}=\left[\frac{\bar{k}}{m \sigma}(1+C)^{p \sigma-m \sigma+1}\right]^{1 /(m \sigma-p \sigma)}
$$

As $m \sigma-p \sigma>0$, we can choose $K$ sufficiently large that $K>$ $K_{1}$ and

$$
[K(\phi(x)+1)]^{\sigma} \geq u_{0}(x) .
$$

Now, it follows from (6)-(8) that $\bar{u}$ defined by (5) is a positive supersolution of (1). Hence $u \leq \bar{u}$ by comparison principle, which implies $u$ exists globally.

\section{Blow-Up Solution to Problem (1)}

In this section, we will discuss the blow-up solution of (1) under some appropriate hypotheses and show Theorem 2 .

Proof. Our strategy here is to construct blow-up subsolutions in some subdomain of $\Omega$ in which $u>0$. Some ideas are borrowed from the work [11] by Du.

Let $\psi(x)$ be a nontrivial nonnegative continuous function and vanish on $\partial \Omega$. Without loss of generality, we assume that $0 \in \Omega$ and $\psi(0)>0$. We will construct a blow-up subsolution to complete the proof.

Set

$$
\underline{u}(x, t)=\frac{1}{(T-t)^{l}} \omega^{1 / m}\left(\frac{|x|}{(T-t)^{\mu}}\right),
$$

with

$$
\omega(r)=\frac{R^{3}}{12}-\frac{R}{4} r^{2}+\frac{1}{6} r^{3}, \quad r=\frac{|x|}{(T-t)^{\mu}}, 0 \leq r \leq R,
$$

where $l, \mu$, and $T>0$ are determined later. Clearly, $0 \leq \omega(r) \leq$ $R^{3} / 12$ and $\omega(r)$ is nonincreasing since $\omega^{\prime}(r)=r(r-R) / 2 \leq 0$. Note that

$$
\operatorname{supp} \underline{u}(\cdot, t)=B\left(0, R(T-t)^{\mu}\right) \subset B\left(0, R T^{\mu}\right) \subset \Omega,
$$

for sufficiently small $T>0$. Obviously, $\underline{u}$ becomes unbounded as $t \rightarrow T^{-}$at the point $x=0$. 
Calculating directly, we obtain

$$
\begin{aligned}
\underline{u}_{t}(x, t)-\Delta \underline{u}^{m}(x, t) \\
=\frac{m l \omega^{1 / m}(r)+\mu r \omega^{\prime}(r) \omega^{(1-m) / m}(r)}{m(T-t)^{l+1}} \\
\quad+\frac{R-2 r}{2(T-t)^{m l+2 \mu}}+\frac{(N-1)(R-r)}{2(T-t)^{m l+\mu}} \\
\leq \frac{l\left(R^{3} / 12\right)^{1 / m}}{(T-t)^{l+1}}+\frac{N R-(N+1) r}{2(T-t)^{m l+2 \mu}},
\end{aligned}
$$

noticing that $T>0$ is sufficiently small.

Case 1. If $0 \leq r \leq N R /(N+1)$, we have $\omega(r) \geq(3 N+$ 1) $R^{3} / 12(N+1)^{3}$; then

$$
\begin{aligned}
f(t) g(\underline{u}) & \geq f(t) \underline{u}^{p}(x, t)=f(t) \frac{1}{(T-t)^{p l}} \omega^{p / m}(r) \\
& \geq \frac{\underline{k}}{(T-t)^{p l}}\left(\frac{(3 N+1) R^{3}}{12(N+1)^{3}}\right)^{p / m} .
\end{aligned}
$$

Hence

$$
\begin{aligned}
& \underline{u}_{t}(x, t)-\Delta \underline{u}^{m}(x, t)-f(t) g(\underline{u}) \\
& \quad \leq \frac{l\left(R^{3} / 12\right)^{1 / m}}{(T-t)^{l+1}}-\frac{\underline{k}}{(T-t)^{p l}}\left(\frac{(3 N+1) R^{3}}{12(N+1)^{3}}\right)^{p / m} .
\end{aligned}
$$

Case 2. If $N R /(N+1)<r \leq R$, then

$$
\begin{aligned}
& \underline{u}_{t}(x, t)-\Delta \underline{u}^{m}(x, t)-f(t) g(\underline{u}) \\
& \leq \frac{l\left(R^{3} / 12\right)^{1 / m}}{(T-t)^{l+1}}+\frac{N R-(N+1) r}{2(T-t)^{m l+2 \mu}} .
\end{aligned}
$$
that

If $p \geq m>1$, then there exists positive constant $l$, such

$$
(m-1) l>1 \text {. }
$$

Thus, we get

$$
\begin{gathered}
p l \geq m l>l+1, \\
m l+2 \mu>l+1 \quad(\forall \mu>0) .
\end{gathered}
$$

Hence, for sufficiently small $\mu>0$ and $T>0$, (11) holds. And (14)-(15) imply that

$$
\underline{u}_{t}(x, t)-\Delta \underline{u}^{m}(x, t)-f(t) g(\underline{u}) \leq 0,
$$

where $(x, t) \in B\left(0, R(T-t)^{\mu}\right) \times(0, T)$.

Since $\psi(x)$ is a nontrivial nonnegative continuous function and $\psi(0)>0$, there exist two positive constants $\rho$ and $\varepsilon$ such that $\psi(x)>\varepsilon$ for all $x \in B(0, \rho) \subset \Omega$. Choose $T$ small enough to insure $B\left(0, R(T-t)^{\mu}\right) \subset B(0, \rho)$; hence $\underline{u} \leq 0$ on $\partial B\left(0, R(T-t)^{\mu}\right) \times(0, T)$. From (11), it follows that $\underline{u}(x, 0) \leq \bar{M} \psi(x)$ for sufficient large $\bar{M}$. By comparison principle, we have $\underline{u} \leq u$ provided that $u_{0}(x) \geq \bar{M} \psi(x)$, which implies that the solution $u$ of (1) blows up in finite time.

\section{Upper Bound for Blow-Up Time}

In this section, we will discuss upper bound for the blowup time under some appropriate hypotheses and show Theorem 3.

Proof. Denote

$$
F(t)=\int_{\Omega} u \varphi d x
$$

where $\varphi$ is the solution to problem (2).

Case 1 $(p>m>1)$. Combining (1) and (19), we have

$$
\begin{aligned}
F^{\prime}(t) & =\int_{\Omega}\left[\Delta u^{m}+f(t) g(u)\right] \varphi d x \\
& \geq-\lambda_{1} \int_{\Omega} u^{m} \varphi d x+\underline{k} \int_{\Omega} u^{p} \varphi d x .
\end{aligned}
$$

As $p>m>1$, making use of Hölder's and Young's inequality, we have

$$
\begin{aligned}
& \int_{\Omega} u^{m} \varphi d x \\
& \leq\left(\int_{\Omega} u \varphi d x\right)^{(p-m) /(p-1)}\left(\int_{\Omega} u^{p} \varphi d x\right)^{(m-1) /(p-1)} \\
& \quad \leq \frac{p-m}{p-1} \int_{\Omega} u \varphi d x+\frac{m-1}{p-1} \int_{\Omega} u^{p} \varphi d x \\
& \int_{\Omega} u^{p} \varphi d x \geq\left(\int_{\Omega} u \varphi d x\right)^{p} .
\end{aligned}
$$

Applying (19)-(21), we obtain the inequality

$$
F^{\prime}(t) \geq-A_{1} F(t)+A_{2} F^{p}(t),
$$

where $A_{1}=\lambda_{1}((p-m) /(p-1)), A_{2}=\underline{k}-\lambda_{1}((m-1) /(p-1))>$ 0 .

Choosing $u_{0}(x)$ sufficiently large such that

$$
A_{2} F^{p-1}(0)-A_{1}>0
$$

we conclude that $F(t)$ is increasing monotonously for any $t>$ 0 .

Moreover, according to (22) and $p>1$, we can obtain that there exists $T_{1}>0$, such that

$$
\begin{aligned}
\lim _{t \rightarrow T_{1}^{-}} F(t) & =+\infty, \\
T_{1} & \leq \int_{F(0)}^{+\infty} \frac{d \tau}{-A_{1} \tau+A_{2} \tau^{p}}<+\infty,
\end{aligned}
$$

where $F(0)=\int_{\Omega} u_{0} \varphi d x$.

Case $2(p=m>1)$. According to (1) and (19), we can obtain

$$
\begin{aligned}
F^{\prime}(t) & =\int_{\Omega}\left[\Delta u^{m}+f(t) g(u)\right] \varphi d x \\
& \geq\left(\underline{k}-\lambda_{1}\right) \int_{\Omega} u^{p} \varphi d x .
\end{aligned}
$$


Moreover, applying $p>1$, we have

$$
\int_{\Omega} u^{p} \varphi d x \geq\left(\int_{\Omega} u \varphi d x\right)^{p} .
$$

Combining (25) and (26), we obtain the inequality

$$
F^{\prime}(t) \geq A_{3} F^{p}(t)
$$

where $A_{3}=\underline{k}-\lambda_{1}>0$.

Choosing $u_{0}(x)$ sufficiently large yields

$$
F(0)>0
$$

Thus we conclude that $F(t)$ is increasing monotonously for any $t>0$.

Furthermore, according to $p>1$ and (27), we can obtain that there exists $T_{2}>0$, such that

$$
\begin{aligned}
& \lim _{t \rightarrow T_{2}^{-}} F(t)=+\infty, \\
& T_{2} \leq \int_{F(0)}^{+\infty} \frac{d \tau}{A_{3} \tau^{p}}<+\infty .
\end{aligned}
$$

Hence, denote $\bar{T}_{0}=\min \left\{T_{1}, T_{2}\right\}$, which depends on $u_{0}(x), m, f$, and $g$, and Theorem 3 is completed.

\section{Lower Bound for Blow-Up Time}

In this section, we will give the lower bound to the blow-up time as long as blow-up occurs and show Theorem 4 .

Proof. Firstly, according to Theorem 1, we have $p \geq m$ under the conditions of Theorem 4 .

Secondly, set

$$
\Phi(t)=\int_{\Omega} u^{2 p+m+1} d x
$$

We compute

$$
\begin{aligned}
\Phi^{\prime}(t) & \\
= & (2 p+m+1) \int_{\Omega} u^{2 p+m}\left[\Delta u^{m}+f(t) g(u)\right] d x \\
= & -\frac{(2 p+m+1)(2 p+m)}{(p+m)^{2}} \int_{\Omega}\left|\nabla u^{(p+m)}\right|^{2} d x \\
& +(2 p+m+1) f(t) \int_{\Omega} u^{2 p+m} g(u) d x \\
\leq & -\frac{(2 p+m+1)(2 p+m)}{(p+m)^{2}} \int_{\Omega}\left|\nabla u^{(p+m)}\right|^{2} d x \\
& +(2 p+m+1) f(t) \int_{\Omega} u^{2 p+m+p} d x .
\end{aligned}
$$

Clearly, making use of Hölder's inequality, we get

$$
\begin{aligned}
& f(t) \int_{\Omega} u^{2 p+m+p} d x \leq f(t)\left(\int_{\Omega} u^{2(2 p+m)} d x\right)^{1 / 2} \\
& \cdot\left(\int_{\Omega} u^{2 p} d x\right)^{1 / 2} \leq f(t) \\
& \cdot\left(\int_{\Omega} u^{6(p+m)} d x\right)^{(2 p+m-1) / 2(4 p+5 m-1)} \\
& \quad \cdot\left(\int_{\Omega} u^{2 p+m+1} d x\right)^{(p+2 m) /(4 p+5 m-1)}\left(\int_{\Omega} u^{2 p} d x\right)^{1 / 2} .
\end{aligned}
$$

Applying Sobolev's inequality (see [1]) in $\mathbb{R}^{3}$

$$
\left(\int_{\Omega} \zeta^{6} d x\right)^{1 / 6} \leq 4^{1 / 3} 3^{-1 / 2} \pi^{-2 / 3}\left(\int_{\Omega}|\nabla \zeta|^{2} d x\right)^{1 / 2},
$$

we can obtain

$$
\int_{\Omega} u^{6(p+m)} d x \leq \frac{16}{27 \pi^{4}}\left(\int_{\Omega}\left|\nabla u^{(p+m)}\right|^{2} d x\right)^{3} .
$$

Substituting (23) into (22) yields

$$
\begin{aligned}
& f(t) \int_{\Omega} u^{2 p+m+p} d x \leq f(t)\left(\frac{2 \sqrt[3]{2}}{3 \pi \sqrt[3]{\pi}}\right. \\
& \left.\cdot \varepsilon \int_{\Omega}\left|\nabla u^{(p+m)}\right|^{2} d x\right)^{3(2 p+m-1) / 2(4 p+5 m-1)} \\
& \quad \cdot\left\{\varepsilon^{-3(2 p+m-1) /(2 p+7 m+1)}\left(\int_{\Omega} u^{2 p+m+1} d x\right)^{(2 p+4 m) /(2 p+7 m+1)}\right. \\
& \left.\cdot\left(\int_{\Omega} u^{2 p} d x\right)^{(4 p+5 m-1) /(2 p+7 m+1)}\right\}^{(2 p+7 m+1) / 2(4 p+5 m-1)} \\
& \quad \leq \frac{\sqrt[3]{2}(2 p+m-1)}{\pi \sqrt[3]{\pi}(4 p+5 m-1)} \varepsilon \int_{\Omega}\left|\nabla u^{(p+m)}\right|^{2} d x \\
& \quad+\frac{2 p+7 m+1}{2(4 p+5 m-1)} \\
& \cdot \varepsilon^{-3(2 p+m-1) /(2 p+7 m+1)}\left(\int_{\Omega} u^{2 p+m+1} d x\right)^{(2 p+4 m) /(2 p+7 m+1)} \\
& \quad \cdot|\Omega|^{-3(2 p+m-1) /(2 p+7 m+1)}\left(\int_{\Omega} u^{2 p+m+1} d x\right)^{(2 p+4 m) /(2 p+7 m+1)} \\
& \left.\quad \cdot\left[f^{2}(t) \int_{\Omega}^{2 p+m+1} d x\right)^{2 p} d x\right]^{2(4 p+5 m-1) /(2 p+7 m+1)} \\
& \quad \leq \frac{\sqrt[3]{2}(2 p+m-1)}{\pi \sqrt[3]{\pi}(4 p+5 m-1)} \varepsilon \int_{\Omega}\left|\nabla u^{(p+m)}\right|^{2} d x \\
& +\frac{2 p+7 m+1}{2(4 p+5 m-1)}
\end{aligned}
$$

where $\varepsilon=\pi \sqrt[3]{\pi}(2 p+m)(4 p+5 m-1) / \sqrt[3]{2}(p+m)^{2}(2 p+m-1)$. 
Substituting (35) into (31), we obtain

$$
\Phi^{\prime}(t) \leq k(t)\left(\int_{\Omega} u^{2 p+m+1} d x\right)^{\mu_{1}+\mu_{2}}=k(t) \Phi(t)^{\mu_{1}+\mu_{2}},
$$

where

$$
\begin{aligned}
& k(t)=(2 p+m+1) \frac{2 p+7 m+1}{2(4 p+5 m-1)} \\
& \cdot \varepsilon^{-3(2 p+m-1) /(2 p+7 m+1)}|\Omega|^{1-2 p /(2 p+m+1)} \\
& \cdot f(t)^{2(4 p+5 m-1) /(2 p+7 m+1)}>0, \\
& \mu_{1}=\frac{2 p+4 m}{2 p+7 m+1}>0 \\
& \mu_{2}=\frac{2 p(4 p+5 m-1)}{(2 p+m+1)(2 p+7 m+1)}>0 .
\end{aligned}
$$

We suppose that

$$
\lim _{t \rightarrow T^{-}} \Phi(t)=+\infty, \quad(0<T<+\infty),
$$

and then there exists $t_{1}>0$ such that $\Phi(t)>1\left(t>t_{1}\right)$.

Integrating (36) from $t_{1}$ to $T$, we obtain

$$
\int_{1}^{+\infty} \frac{d \tau}{\tau^{\mu_{1}+\mu_{2}}} \leq \int_{t_{1}}^{T} k(t) d t \leq \int_{0}^{T} k(t) d t
$$

Let $\Theta(T)=\int_{0}^{T} k(t) d t$. Obviously, $\Theta(T)$ is increasing monotonously. So we can obtain

$$
T \geq \Theta^{-1}(\theta):=\underline{T}_{0}>0,
$$

where $\theta=\int_{1}^{+\infty}\left(d \tau / \tau^{\mu_{1}+\mu_{2}}\right), \Theta^{-1}$ is the inverse function of $\Theta$, and $\underline{T}_{0}$ depends on $u_{0}(x), m, f$, and $g$.

Thus, we complete the proof of Theorem 4 .

\section{Conflict of Interests}

The authors declare that there is no conflict of interests regarding the publication of this paper.

\section{Acknowledgments}

The authors would like to thank the reviewers for careful reading and giving many helpful comments to improve the paper. This work is supported in part by NSFC Grant (no. 61563044), the Science and Technology Major Project of Qinghai Province Natural Science Foundation (no. 2015-SFA4-3), and SRFDP (no. 20100181110036).

\section{References}

[1] C. Bandle and H. Brunner, "Blow-up in diffusion equations," Journal of Computational and Applied Mathematics, vol. 97, no. 1-2, pp. 3-22, 1998.

[2] F. C. Li and C. H. Xie, "Global existence and blow-up for a nonlinear porous medium equation," Applied Mathematics Letters, vol. 16, no. 2, pp. 185-192, 2003.
[3] P. Quittner and P. Souplet, Superlinear Parabolic Problems: Blow-up, Global Existence and Steady States, Birkhäuser, Basel, Switzerland, 2007.

[4] F. B. Weissler, "Existence and non-existence of global solutions for a semilinear heat equation," Israel Journal of Mathematics, vol. 38, no. 1-2, pp. 29-40, 1981.

[5] L. E. Payne and P. W. Schaefer, "Lower bounds for blow-up time in parabolic problems under Dirichlet conditions," Journal of Mathematical Analysis and Applications, vol. 328, no. 2, pp. 1196-1205, 2007.

[6] L. E. Payne and G. A. Philippin, "Blow-up phenomena in parabolic problems with time dependent coefficients under Dirichlet boundary conditions," Proceedings of the American Mathematical Society, vol. 141, no. 7, pp. 2309-2318, 2013.

[7] L. E. Payne and G. A. Philippin, "Blow-up phenomena for a class of parabolic systems with time dependent coefficients," Applied Mathematics, vol. 3, no. 4, pp. 325-330, 2012.

[8] W. B. Deng, "Global existence and finite time blow up for a degenerate reaction-diffusion system," Nonlinear Analysis. Theory, Methods \& Applications, vol. 60, no. 5, pp. 977-991, 2005.

[9] L. L. Du, C. L. Mu, and M. S. Fan, "Global existence and nonexistence for a quasilinear degenerate parabolic system with non-local source," Dynamical Systems, vol. 20, no. 4, pp. 401412, 2005.

[10] L. L. Du, "Blow-up for a degenerate reaction-diffusion system with nonlinear localized sources," Journal of Mathematical Analysis and Applications, vol. 324, no. 1, pp. 304-320, 2006.

[11] L. L. Du, "Blow-up for a degenerate reaction-diffusion system with nonlinear nonlocal sources," Journal of Computational and Applied Mathematics, vol. 202, no. 2, pp. 237-247, 2007.

[12] L. L. Du and C. L. Mu, "Global existence and blow-up analysis to a degenerate reaction-diffusion system with nonlinear memory," Nonlinear Analysis: Real World Applications, vol. 9, no. 2, pp. 303-315, 2008.

[13] L. L. Du and Z. Y. Xiang, "A further blow-up analysis for a localized porous medium equation," Applied Mathematics and Computation, vol. 179, no. 1, pp. 200-208, 2006.

[14] L. L. Du and Z.-A. Yao, "Localization of blow-up points for a nonlinear nonlocal porous medium equation," Communications on Pure and Applied Analysis, vol. 6, no. 1, pp. 183-190, 2007.

[15] M. S. Fan, C. L. Mu, and L. L. Du, "Uniform blow-up profiles for a nonlocal degenerate parabolic system," Applied Mathematical Sciences, vol. 1, no. 1-4, pp. 13-23, 2007.

[16] E. DiBenedetto, Degenerate Parabolic Equations, Universitext, Springer, New York, NY, USA, 1993. 


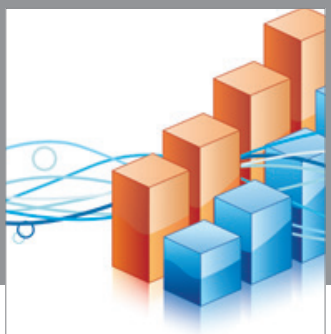

Advances in

Operations Research

vatem alat4

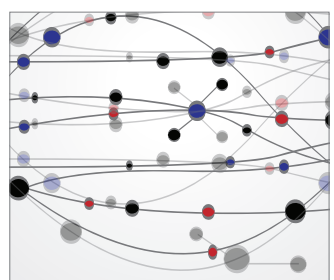

\section{The Scientific} World Journal
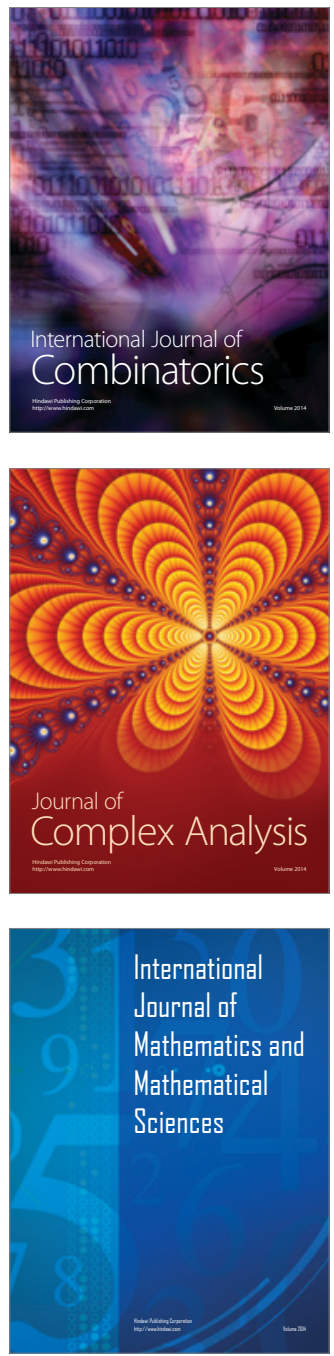
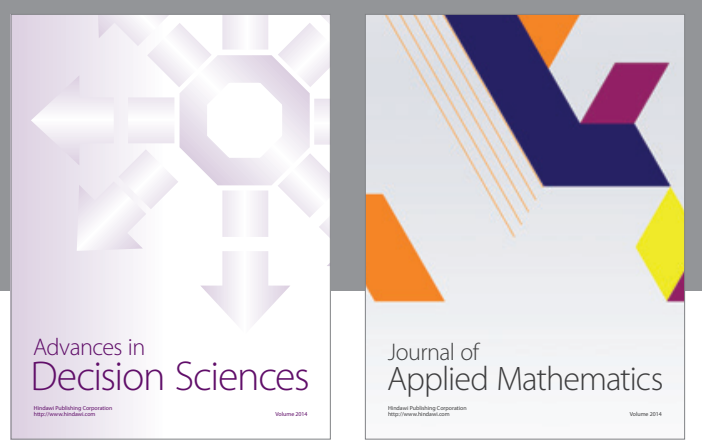

Algebra

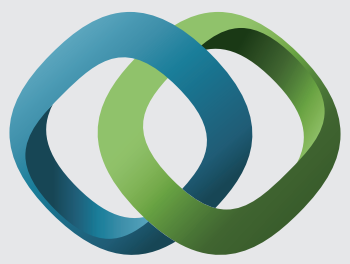

\section{Hindawi}

Submit your manuscripts at

http://www.hindawi.com
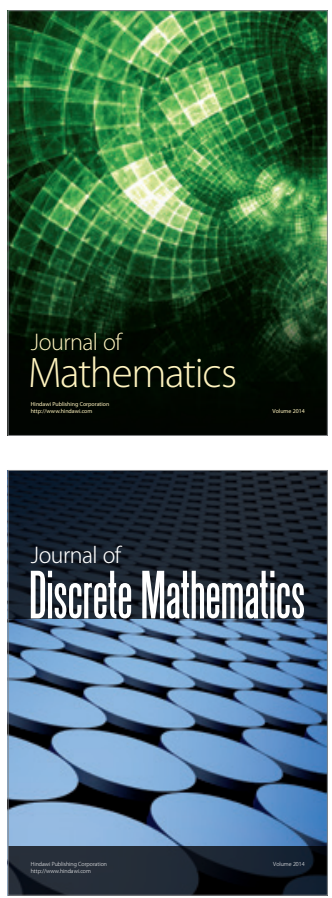

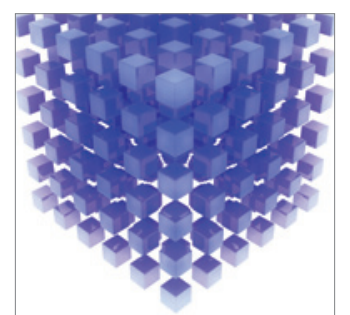

Mathematical Problems in Engineering
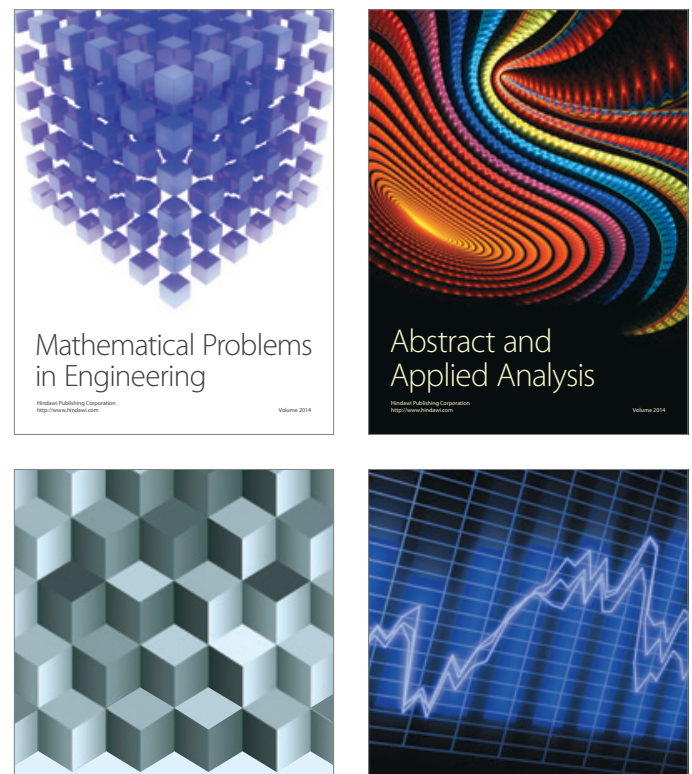

Journal of

Function Spaces

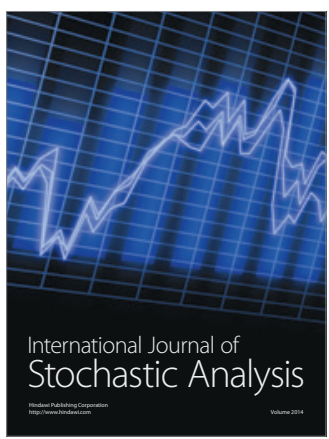

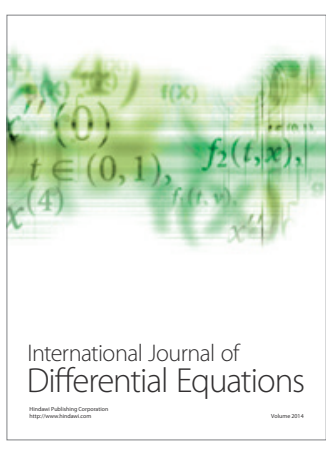
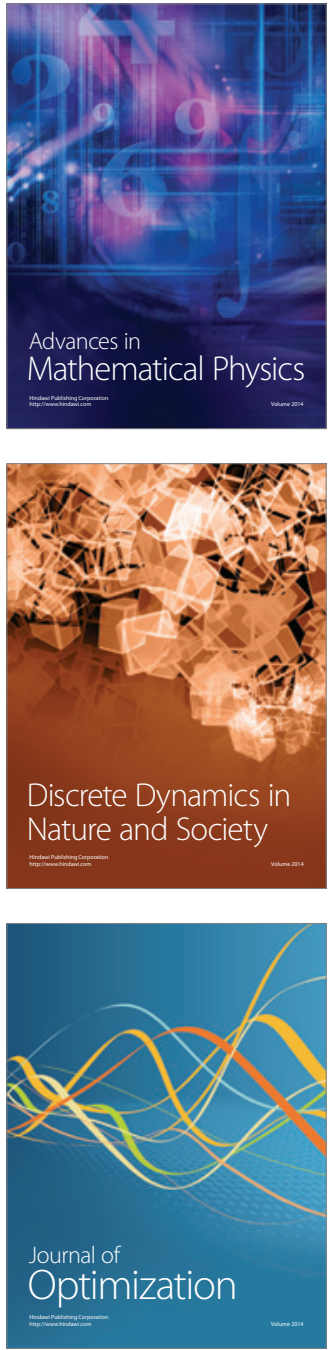